\title{
The new Wolf-Rayet binary system WR62a
}

\author{
A. Collado ${ }^{1, \star}$, R. Gamen ${ }^{2, \star \star}$, and R. H. Barbá ${ }^{1,3, \star \star \star}$ \\ ${ }^{1}$ Instituto de Ciencias Astronómicas de la Tierra y del Espacio (ICATE), CONICET, Avda. España 1512 Sur, J5402DSP, San Juan, \\ Argentina \\ 2 Instituto de Astrofísica de La Plata, CONICET; Facultad de Ciencias Astronómicas y Geofísicas, Universidad Nacional de La Plata, \\ Paseo del bosque s/n, B1900FWA, La Plata, Argentina \\ e-mail: rgamen@fcaglp.unlp.edu.ar \\ 3 Departamento de Física, Universidad de La Serena, Av. Cisternas 1200 Norte, La Serena, Chile \\ e-mail: acollado@icate-conicet.gob.ar
}

Received 15 November 2011 / Accepted 16 January 2013

\section{ABSTRACT}

\begin{abstract}
Context. A significant number of the Wolf-Rayet stars seem to be binary or multiple systems, but the nature of many of them is still unknown. Dedicated monitoring of WR stars favours the discovery of new systems.

Aims. We explore the possibility that WR62a is a binary system.

Methods. We analysed the spectra of WR62a, obtained between 2002 and 2010, to look for radial-velocity and spectral variations that would suggest there is a binary component. We searched for periodicities in the measured radial velocities and determined orbital solutions. A period search was also performed on the "All-Sky Automated Survey" photometry.

Results. We find that WR62a is a double-lined spectroscopic binary with a WN5 primary star and an O 5.5-6 type secondary component in orbit with a period of $9.1447 \mathrm{~d}$. The minimum masses range between 21 and $23 M_{\odot}$ for the WN star and between 39 and $42 M_{\odot}$ for the O-type star, thus indicating that the WN star is less massive than the O-type component. We detect a phase shift in the radial-velocity curve of the He II $\lambda 4686$ emission line relative to the other emission line curves. The equivalent width of this emission line shows a minimum value when the WN star passes in front of the system. The analysis of the ASAS photometry confirms the spectroscopic periodicity, presenting a minimum at the same phase.
\end{abstract}

Key words. binaries: spectroscopic - stars: individual: SMSNPL11 (=WR62a) - stars: Wolf-Rayet - stars: fundamental parameters

\section{Introduction}

Wolf-Rayet stars frequently occur in binary systems. In fact, multiplicity seems to be one of the most striking properties of massive stars (cf. Sana \& Evans 2011). van der Hucht (2001) has found $38 \%$ binaries and possible binaries among a total of 227 WR stars. Langer \& Heger (1999) argue that most of the Supernova Type Ib/c occur in WR + OB interacting binary systems, and given the statistics derived from the SN observed, it would be expected that more WR stars are detected in binary systems than as single stars. Another reason is provided by the observation of late-type WC stars (WC8-9). These stars exhibit thermal emission from hot dust, and it is generally accepted that the production of dust is a consequence of the binarity, where the dust is created via the collision of hydrogen-rich and carbon-rich winds from the respective OB and WC stars (Usov 1991). Moreover, a systematic high-resolution spectroscopic survey of O and WN stars (OWN Survey; Barbá et al. 2010) has found that nearly $60 \%$ of the sample are candidates to belong in binary or multiple systems. Therefore, the binary fraction is expected to be higher than what was found by van der Hucht, that is, the binarity appears to play a key role in the

* Fellow of CONICET, and visiting astronomer, Cerro Tololo InterAmerican Observatory (Chile) and CASLEO (Argentina).

$\star \star$ Visiting astronomer, Cerro Tololo Inter-American Observatory (Chile) and CASLEO (Argentina).

$\star \star \star$ Visiting astronomer, Las Campanas Observatory and Cerro Tololo Inter-American Observatory, Chile. formation of WR stars. In recent years, the discovery of new binary systems has been favoured thanks to technological advances, allowing us to understand a little more about the nature of massive stars.

The stellar mass is a fundamental astrophysical parameter whose knowledge, together with the rate of mass loss, chemical composition, and rotation, determines the properties and evolution of stars (Meynet \& Maeder 2005). Observing binaries, particularly double-lined spectroscopic binary systems (SB2) that are eclipsing, is the most direct way to obtain accurate stellar masses.

We are carrying out spectroscopic monitoring of faint southern Galactic Wolf-Rayet stars in order to discover new binary systems. One of the most conspicuous radial-velocity (RV) variables observed in our sample is WR62a. This star (SMSNPL11, $\left.\alpha_{2000}=14^{\mathrm{h}} 32^{\mathrm{m}} 38^{\mathrm{s}} .18 ; \delta_{2000}=-61^{\circ} 29^{\prime} 55^{\prime \prime} .4 ; v=13.8\right)$ was discovered by Shara et al. (1999). They classified it as WN4-5o, which means that they did not identify absorption lines in the spectrum.

In this paper, we present the first detailed spectral analysis for WR62a. We demonstrate that it is a short-period SB2. We also report a phase shift in the orbital solution of one of its emission lines and discuss the photometric variability of the star.

\section{Observations and data reduction}

We have obtained a total of 28 spectral images of WR62a. The images were acquired with the 4-m V. Blanco telescope at Cerro 
Table 1. Details of the observations.

\begin{tabular}{lccccccccc}
\hline \hline $\begin{array}{l}\text { Date-Obs. } \\
\text { UT }\end{array}$ & $n$ & $\begin{array}{c}\text { Sp. coverage } \\
{[\AA]}\end{array}$ & Observat. & $\begin{array}{c}\text { Telesc. } \\
{[\mathrm{m}]}\end{array}$ & Spectr. $^{a}$ & $\begin{array}{c}\text { Grating }^{\circ} \\
{\left[\mathrm{mm}^{-1}\right]}\end{array}$ & $\begin{array}{c}\text { Detector } \\
{\left[\AA \text { pix }^{-1}\right]}\end{array}$ & $\begin{array}{c}\text { Dispersion } \\
\text { Resolution }^{b}\end{array}$ \\
\hline 2002, Apr. 22 & 1 & $3940-5600$ & CASLEO & 2.15 & REOSC & 600 & Tek1024 & 1.63 & 1000 \\
2007, Mar. 29-Apr. 3 & 6 & $3650-6700$ & CTIO & 4 & R-C & 632 & Loral 3k & 1.01 & 1300 \\
2008, Apr. 19-23 & 6 & $3650-6700$ & CTIO & 4 & R-C & 632 & Loral 3k & 1.01 & 1300 \\
2009, Mar. 24-27 & 4 & $4030-5590$ & CASLEO & 2.15 & REOSC & 600 & Tek1024 & 1.63 & 1000 \\
2009, Apr. 20-21 & 2 & $3800-5530$ & LCO & 2.5 & B-C & 1200 & Marconi\#1 & 0.79 & 2500 \\
2009, Jul. 19-27 & 4 & $3520-5250$ & LCO & 2.5 & B-C & 1200 & Marconi\#1 & 0.79 & 2500 \\
2009, Aug. 14-16 & 3 & $4040-5700$ & CASLEO & 2.15 & REOSC & 600 & Tek1024 & 1.63 & 1000 \\
2010, Apr. 10-13 & 2 & $3930-5600$ & CASLEO & 2.15 & REOSC & 600 & Tek1024 & 1.63 & 1000 \\
\hline
\end{tabular}

Notes. $n$ : Number of spectra obtained per run. ${ }^{(a)}$ Details of the spectrographs can be found in the User's Manuals of the respective Observatories. (b) The spectral resolutions $(R=\lambda / \Delta \lambda)$ were measured using $\Delta \lambda$ as the FWHM of the calibration lamp emission lines.

Tololo Inter-American Observatory (CTIO), Chile; the 2.5-m du Pont telescope at Las Campanas Observatory (LCO), Chile; and the 2.15-m J. Sahade telescope at Complejo Astronómico El Leoncito (CASLEO) ${ }^{1}$, Argentina, between 2002 and 2010. See Table 1 for a summary of the observations. We have acquired spectra at CTIO using the Ritchey-Chretien (R-C) spectrograph, at CASLEO employing the REOSC spectrograph, and at LCO using a Boller \& Chivens (B-C) spectrograph. The procedure for obtaining LCO spectra are also described in detail by Sota et al. (2011). Slight differences in the spectral ranges listed in Table 1 are due to changes in the grating angle among the observing runs.

Typical exposure times for spectra were between 15 and $40 \mathrm{~min}$, resulting in spectra with signal-to-noise ratios $S / N \sim 50-150$. Comparison lamp spectra of $\mathrm{He}-\mathrm{Ne}-\mathrm{Ar}$ (or $\mathrm{Cu}-$ Ar with REOSC spectrograph) were observed immediately after or before, at the same telescope position as the stellar exposures. Bias and flat-field images were also obtained each night. We did not observe a telluric standard star since there are not many atmospheric absorption lines in the wavelength range of our spectra. All spectra were processed with IRAF ${ }^{2}$ routines.

\section{Results and discussion}

\subsection{The spectrum of WR $62 a$}

Our spectra reveal that WR62a is a double-lined WR+OB binary system. Several absorption lines are detected in our spectra. The spectra obtained at different epochs show that the absorption lines have different radial-velocity shifts from those of the emission lines, suggesting that the system is an SB2. We used an iterative procedure similar to the one developed by Marchenko et al. (1998) to separate the composite spectrum into the spectra from the two components of the binary. First, we begin by shifting the spectra to its respective radial velocity and calculating the mean spectrum to be used as a template of the WN. Second, the RV-shifted mean WR spectrum is subtracted from the original spectra, obtaining the remaining O-type spectra. Then, they are shifted to their corresponding RV of the absorption lines and combined to get the template of the O-type star. After that, the

\footnotetext{
1 Operated under agreement between CONICET and the Universities of La Plata, Córdoba, and San Juan, Argentina.

2 IRAF is distributed by the National Optical Astronomy Observatories, which are operated by the Association of Universities for Research in Astronomy, Inc., under cooperative agreement with the National Science Foundation.
}

(RV-shifted) mean O-type spectrum is subtracted from the observed spectra and a new purer WN-type spectrum is obtained. That is to say, the spectra are shifted, combined, and subtracted appropriately to compute the individual spectra of the stellar components. We noted that the method converges after a few iterations.

After separating the respective spectra (see Fig. 1), we performed a more detailed spectral classification. Relative intensities of N III, N IV, He II, and C IV emission lines in the WR spectrum indicate a WN5 spectral type (according to the criteria given by Smith et al. 1996), confirming the classification proposed by Shara et al. (1999).

In the disentangled spectrum of the O-type star, we identified absorption lines of $\mathrm{H} \alpha, \mathrm{H} \beta, \mathrm{H} \gamma, \mathrm{H} \delta$, He I $\lambda \lambda$ 4026, 4471, and 5875, and also He II $\lambda \lambda$ 4200, 4542, 4686, and 5411, as well as the C III 5696 emission line. The comparison between the He I $\lambda 4471$ and the He II $\lambda 4542$ absorption lines indicates that the O-type star is earlier than $\mathrm{O} 7$ spectral type; i.e., the intensity of the former He I line is fainter than that of the He II line. An improved spectral classification was achieved through the comparison of the $\mathrm{O}$ star spectrum with the MK standards from the new Atlas for spectral classification (Sota et al. 2011). Thus, an O 5.5-6 type is determined. A luminosity class is not possible to determine owing to the profile variations in the emission lines of the WN-type component which introduce residuals in the resulting O-type spectrum (for example around 4600-4700 ̊).

\subsection{Analysis of radial velocities}

We have determined the RVs of the WN component of WR62a by measuring the position of the stronger emission lines, i.e. $\mathrm{N}$ IV $\lambda 4058, \mathrm{~N} \mathrm{~V} \lambda 4604$, and He II $\lambda \lambda 4200,4542,4686$, and 5411. For the O-type star, we determined a mean RV value for each spectrum by averaging the RV measurements derived from the absorption lines. These measurements are listed in Table 2.

The strongest emission line in our spectra corresponds to He II $\lambda 4686$, so we could measure this line even in spectra with poor signal-to-noise ratios. The RVs of this line show large variations from night to night, suggesting an orbital period of a few days. We have searched for periodicities by means of the algorithm published by Marraco \& Muzzio (1980). This method divides the observations into several phase intervals and computes the variance with respect to the best-fitting straight line in each interval. The most probable period obtained corresponds to $9.144 \mathrm{~d}$ (Fig. 2), and it is used as initial value to feed orbital solutions for the RV data. 
A. Collado et al.: The new Wolf-Rayet binary system WR62a

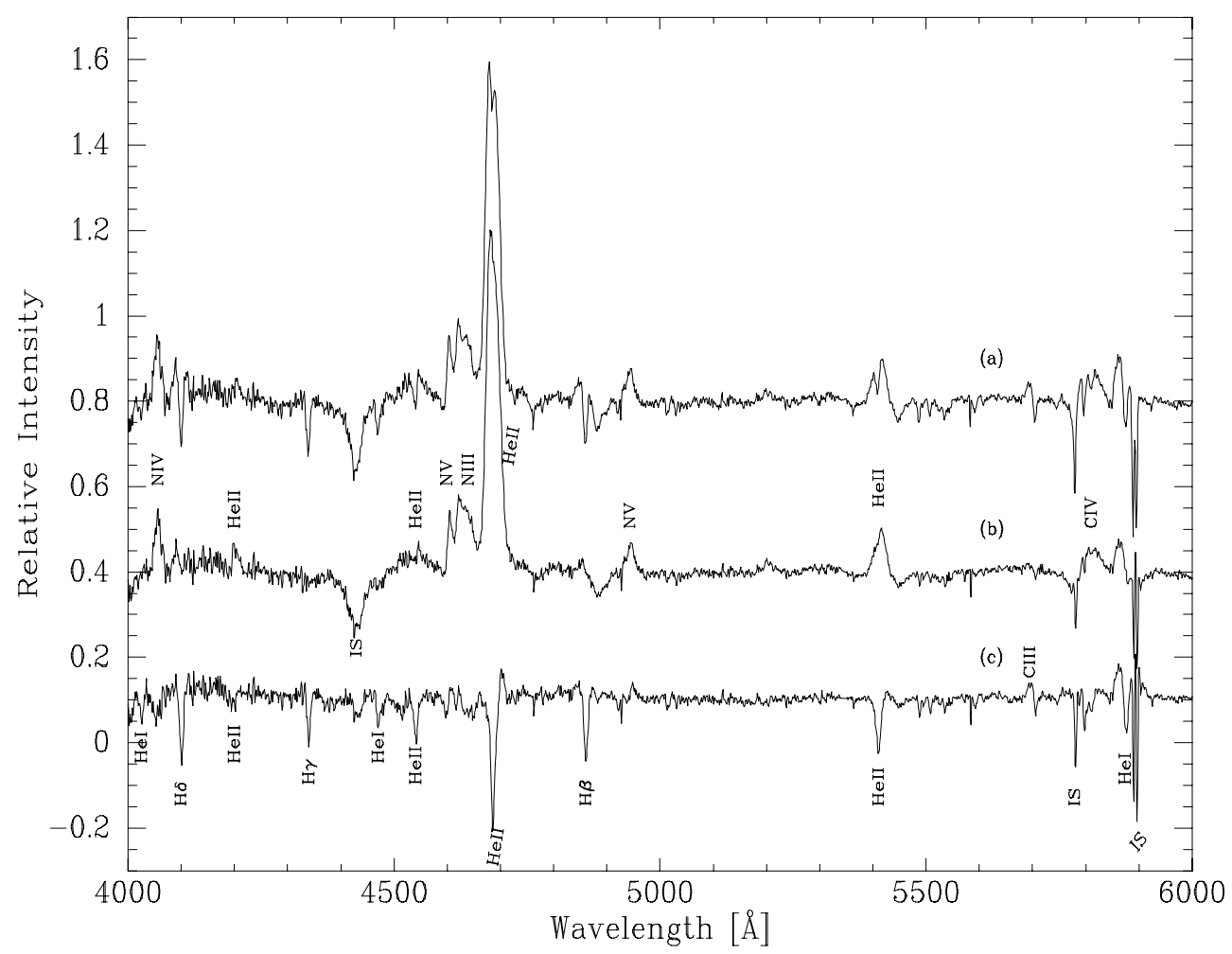

Fig. 1. a) Normalized spectrum of WR62a before the "disentangling" method; b) the WR star component after removing the O-type star; c) the remaining spectrum of the O-type star. The spectra are shifted in the intensity axis for a better comparison.

Table 2. Observed heliocentric radial velocities of WR62a.

\begin{tabular}{|c|c|c|c|c|c|c|c|c|c|c|}
\hline $\begin{array}{l}\text { HJD } \\
2450000+\end{array}$ & Phase & $\begin{array}{c}\text { N IV } \\
4057.66 \AA \\
{\left[\mathrm{km} \mathrm{s}^{-1}\right]} \\
\end{array}$ & $\begin{array}{c}\mathrm{N} \mathrm{V} \\
4603.73 \AA \\
{\left[\mathrm{km} \mathrm{s}^{-1}\right]} \\
\end{array}$ & $\begin{array}{c}\mathrm{N} \mathrm{V} \\
4944.56 \AA \\
{\left[\mathrm{km} \mathrm{s}^{-1}\right]} \\
\end{array}$ & $\begin{array}{c}\text { C IV } \\
5811.98 \AA \\
{\left[\mathrm{km} \mathrm{s}^{-1}\right]} \\
\end{array}$ & $\begin{array}{c}\text { He II } \\
4685.68 \AA \\
{\left[\mathrm{km} \mathrm{s}^{-1}\right]} \\
\end{array}$ & $\begin{array}{c}\text { He II } \\
5411.52 \AA \\
{\left[\mathrm{km} \mathrm{s}^{-1}\right]} \\
\end{array}$ & $\begin{array}{c}\text { He II } \\
4199.87 \AA \\
{\left[\mathrm{km} \mathrm{s}^{-1}\right]} \\
\end{array}$ & $\begin{array}{c}\text { He II } \\
4541.59 \AA \\
{\left[\mathrm{km} \mathrm{s}^{-1}\right]} \\
\end{array}$ & $\begin{array}{c}\text { Mean Abs }[n]^{a} \\
{\left[\mathrm{~km} \mathrm{~s}^{-1}\right]} \\
\end{array}$ \\
\hline 2386.72 & 0.98 & -213 & 49 & & & 113 & 55 & & & $-43 \pm 27(5)$ \\
\hline 4188.89 & 0.05 & -65 & & -41 & 59 & 254 & 235 & & & $-113 \pm 22(8)$ \\
\hline 4189.82 & 0.15 & 53 & 220 & 106 & 155 & 278 & 306 & 308 & 387 & $-204 \pm 32(10)$ \\
\hline 4191.84 & 0.37 & -8 & 213 & 96 & 185 & 134 & 223 & 297 & 397 & $-175 \pm 29(10)$ \\
\hline 4192.85 & 0.48 & -161 & 9 & -8 & -16 & -61 & 86 & 162 & 261 & $-83 \pm 22(9)$ \\
\hline 4193.65 & 0.57 & -275 & -119 & -199 & -155 & -162 & -62 & 42 & -30 & $19 \pm 59(9)$ \\
\hline 4193.87 & 0.59 & -228 & -143 & -280 & -135 & -250 & -192 & -72 & & $18 \pm 52(9)$ \\
\hline 4575.84 & 0.36 & 87 & 187 & 102 & 182 & 133 & 259 & 374 & 359 & $-157 \pm 36(10)$ \\
\hline 4576.82 & 0.47 & -122 & 48 & -32 & -19 & -53 & 79 & 90 & & $-85 \pm 28(6)$ \\
\hline 4577.73 & 0.57 & -285 & -116 & -193 & -178 & -199 & -119 & & -14 & $19 \pm 38(9)$ \\
\hline 4578.80 & 0.69 & -403 & -254 & -346 & -257 & -282 & -284 & & & \\
\hline 4579.59 & 0.77 & -379 & -255 & -276 & -257 & -191 & -234 & -246 & -215 & $31 \pm 37(9)$ \\
\hline 4579.85 & 0.80 & & -257 & & -290 & -173 & -250 & -262 & -230 & $36 \pm 35(9)$ \\
\hline 4914.83 & 0.43 & -8 & 173 & 101 & & 49 & 130 & & 307 & $-75 \pm 26(5)$ \\
\hline 4915.82 & 0.54 & -134 & -14 & -161 & & -89 & -25 & 114 & 139 & $25 \pm 18$ \\
\hline 4916.78 & 0.65 & -255 & & & & -251 & -208 & -181 & -86 & $98 \pm 14(4)$ \\
\hline 4917.74 & 0.75 & -406 & -216 & -343 & & -183 & -282 & & -212 & $91 \pm 23(5)$ \\
\hline 4941.57 & 0.36 & 91 & 254 & 150 & & 218 & 278 & 375 & 362 & $-151 \pm 24(7)$ \\
\hline 4942.71 & 0.48 & -163 & 19 & -97 & & -12 & -21 & 166 & & $-77 \pm 28(8)$ \\
\hline 5031.64 & 0.21 & 81 & 273 & 155 & & 301 & 302 & 326 & 429 & $-204 \pm 21(8)$ \\
\hline 5036.59 & 0.75 & -372 & & -288 & -212 & -168 & -227 & -249 & -205 & $102 \pm 24(7)$ \\
\hline 5038.58 & 0.97 & -204 & -41 & -167 & -185 & 131 & 33 & -32 & 109 & $-97 \pm 36(3)$ \\
\hline 5039.61 & 0.08 & -31 & 123 & -1 & & 263 & 218 & 228 & 244 & $-168 \pm 33(7)$ \\
\hline 5057.52 & 0.04 & & 170 & -118 & & 352 & 314 & & & $-137 \pm 40(7)$ \\
\hline 5059.52 & 0.25 & & 287 & 175 & & 356 & & & & $-183 \pm 28(6)$ \\
\hline 5060.49 & 0.36 & & 191 & 60 & & 187 & 237 & & & $-151 \pm 17(7)$ \\
\hline 5296.79 & 0.20 & 153 & & & & 267 & & & 373 & $-195 \pm 33(6)$ \\
\hline 5299.66 & 0.51 & & & & & -113 & & & & $31 \pm 1(4)$ \\
\hline$F W H M[\AA]$ & & 18.5 & 10.4 & 19.7 & 33.9 & 24.7 & 22.4 & 17.3 & 18.7 & \\
\hline$E W[\AA]$ & & -4.6 & -1.6 & -1.5 & -2.2 & -26.1 & -3.8 & -1.2 & -1.6 & \\
\hline
\end{tabular}

Notes. The mean FWHM and mean EW of each emission line measured from the separated spectra are indicated in the last two rows, respectively. (a) The errors are the standard deviation of the mean, and the $(n)$ values indicate the number of absorption lines measured. 


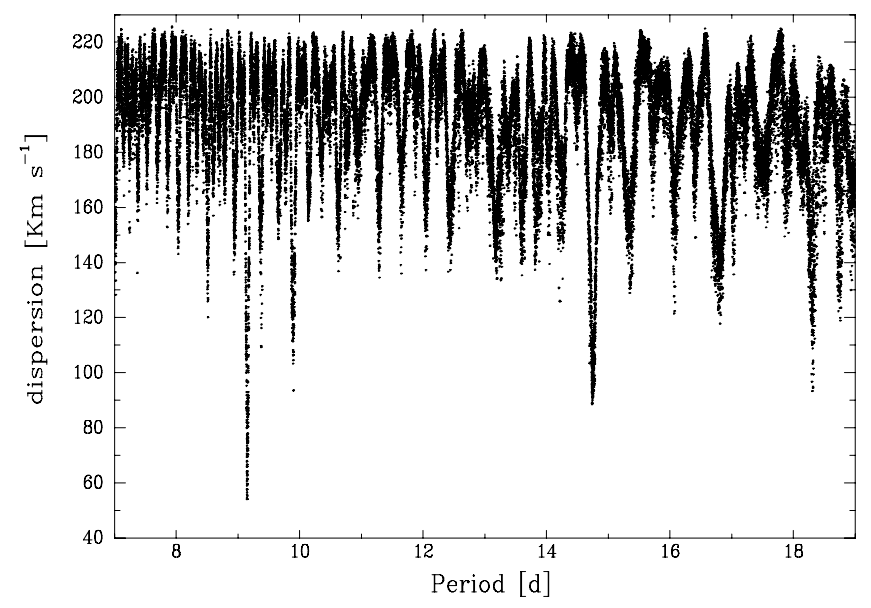

Fig. 2. Periodogram obtained for the He II $\lambda 4686$ emission line with the Marraco \& Muzzio (1980) code. The dispersion ( $Y$-axis) is the parameter used to estimate the quality of the fitting for each trial period. This value is the total variance of the best-fitting straight line to the observations in each phase interval.

Table 3. Periodicities derived from the radial velocity of N IV, N v, C IV, He II emission lines and the mean absorption.

\begin{tabular}{lrl}
\hline \hline Feature & $P[\mathrm{~d}]$ & Error [d] \\
\hline N IV $\lambda 4058$ & 9.1444 & 0.0005 \\
N $\lambda 4604$ & 9.1455 & 0.0005 \\
N $\lambda 4944$ & 9.1443 & 0.0009 \\
C IV $\lambda 5812$ & 9.146 & 0.002 \\
He II $\lambda 4686$ & 9.1447 & 0.0005 \\
He II $\lambda 5411$ & 9.1447 & 0.0003 \\
He II $\lambda 4200$ & 9.145 & 0.001 \\
He II $\lambda 4542$ & 9.140 & 0.002 \\
Mean Abs. & 9.1415 & 0.0005 \\
\hline
\end{tabular}

We performed orbital fits to each dataset of RVs, using the GBART $^{3}$ program. It is clear that the determined RVs for the different spectral features show the same periodicity (see Table 3), and thus, we adopted the straight mean of the periods as the definitive value for the orbital period. The ephemeris adopted for WR62a is

$T_{0}=2455038.9+9.1447 d \times E$

where $T_{0}$ is the time when the WN star passes in front of the system, as in the normal convention for eclipsing WR+OB systems. Then, we calculated the orbital solutions with the period fixed to this average. The orbital parameters derived for each RV dataset are presented in Table 4 (with their corresponding probable errors). To determine the $T_{0}$ of the system, we neglected the very low eccentricity, averaging all the $T_{\mathrm{RV} \max }$ values of the emission lines of Table 4 and calculating the $T_{0}$ as $\left\langle T_{\mathrm{RV} \max }\right\rangle+0.75 \times P$, which coincides with the primary minimum of the lightcurve (as will be shown in Sect. 3.4). Some orbital solutions are illustrated in Fig. 3. The RV curves are phased with the ephemeris from Eq. (1).

In Fig. 3 and Table 4, a phase shift between the He II $\lambda 4686$ line and other emission lines is noticeable. As a result, the $T_{\mathrm{RV} m a x}$ value for this line was excluded from the calculus of the ephemeris. This phase-shift effect is also detected in other close WR binary systems, e.g. HD 90657 (WR 21; Niemela \& Moffat 1982; Gamen 2004), HD 94546 (WR 31; Gamen 2004), and

\footnotetext{
3 GBART is an improved version of the program originally written by Bertiau \& Grobben (1968) and developed by Federico Bareilles.
}

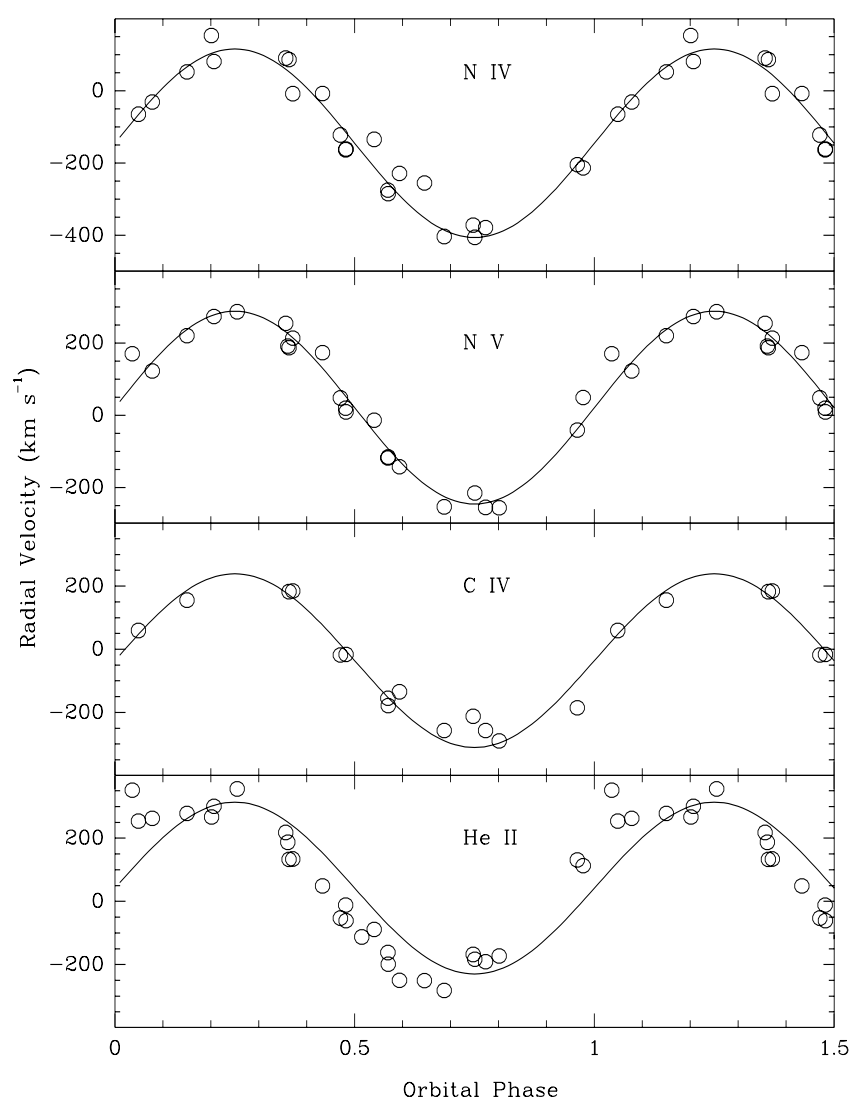

Fig. 3. Radial velocities of N IV $\lambda 4058, \mathrm{~N} v \lambda 4604$, C IV $\lambda 5812$, and He II $\lambda 4686$ emission lines phased with the ephemeris of Eq. (1). Continuous curves represent the orbital solutions from Table 4. The phase shift of the He II line is noticeable.

HDE 320102 (WR 97; Niemela et al. 1995; Gamen 2004), and usually interpreted as a sign of colliding winds. The amplitudes and systemic velocities of the orbital motion determined from the RV of different emission lines show differences. The amplitude of N V $\lambda 4944$ shows the lowest value, while He II $\lambda \lambda 4200$, 4541, and 5411 emission lines appear somewhat larger. The systemic velocities of emission lines (except N IV $\lambda 4058$ and $\mathrm{N} v$ 14944) are redshifted relative to that of the absorption lines. This is a known phenomenon in $\mathrm{WR}+\mathrm{OB}$ systems, which indicates that emission lines are being formed in different regions of the WN wind (cf. Niemela \& Moffat 1982; Niemela et al. 1995), or perhaps are still affected by the absorption lines of the secondary.

From Table 4 and Fig. 4 it is also evident that the absorption lines (the mean of $\mathrm{H}, \mathrm{He}$, He II) move in anti-phase with the emissions, thus they belong to a secondary component. Besides, the semi-amplitude of the orbit of this companion is lower than the WN one (regardless of emission line used), which means that the secondary is more massive than the WN component.

We performed fits of the orbital solution for both components together, by means the GBART program. It is widely known that the RVs of the different emission lines do not strictly follow the same orbital motion in WR binary systems, which is often attributed to their different line-forming regions. We assumed that the orbital motion of the WN star is better represented by the RVs of the highest ionization emission lines, namely, N IV $\lambda 4058 \AA$ and N V $\lambda 4604 \AA$. The O-type star is depicted by the RVs of the mean of some absorption lines. To perform the 
Table 4. The orbital parameters (with their respective probable errors) derived from the radial velocity curves of N IV, N v, C IV, He II emission lines, and the mean absorption.

\begin{tabular}{|c|c|c|c|c|c|c|c|c|c|}
\hline Parameter & $\begin{array}{l}\text { N IV } \\
4058\end{array}$ & $\begin{array}{c}\mathrm{N} \mathrm{V} \\
4604\end{array}$ & $\begin{array}{c}\mathrm{NV} \\
4944\end{array}$ & $\begin{array}{c}\text { C IV } \\
5812\end{array}$ & $\begin{array}{l}\text { He II } \\
4686\end{array}$ & $\begin{array}{l}\text { He II } \\
5411\end{array}$ & $\begin{array}{l}\text { He II } \\
4200\end{array}$ & $\begin{array}{l}\text { He II } \\
4542\end{array}$ & Mean Abs. \\
\hline$P[\mathrm{~d}]$ & & & & & $9.1447 \pm 0.0014$ & & & & \\
\hline$V_{0}\left[\mathrm{~km} \mathrm{~s}^{-1}\right]$ & $-145 \pm 5$ & $21 \pm 5$ & $-94 \pm 5$ & $-36 \pm 7$ & $42 \pm 4$ & $47 \pm 4$ & $80 \pm 6$ & $133 \pm 6$ & $-65 \pm 3$ \\
\hline$K\left[\mathrm{~km} \mathrm{~s}^{-1}\right]$ & $261 \pm 7$ & $267 \pm 7$ & $246 \pm 7$ & $275 \pm 13$ & $272 \pm 6$ & $303 \pm 5$ & $321 \pm 8$ & $320 \pm 8$ & $143 \pm 5$ \\
\hline$e$ & $0.07 \pm 0.02$ & $0.05 \pm 0.03$ & $0.08 \pm 0.02$ & $0.11 \pm 0.04$ & $0.07 \pm 0.02$ & $0.09 \pm 0.02$ & $0.08 \pm 0.02$ & $0.11 \pm 0.03$ & $0.08 \pm 0.04$ \\
\hline$\omega[\mathrm{deg}]$ & $12 \pm 22$ & $160 \pm 29$ & $24 \pm 20$ & $-14 \pm 17$ & $159 \pm 16$ & $183 \pm 11$ & $166 \pm 19$ & $160 \pm 14$ & $319 \pm 21$ \\
\hline$T_{\mathrm{RV} \max }[\mathrm{d}]^{*}$ & $5032.1 \pm 0.5$ & $5032.0 \pm 0.7$ & $5032.2 \pm 0.5$ & $5032.1 \pm 0.4$ & $5031.4 \pm 0.4$ & $5031.8 \pm 0.3$ & $5032.3 \pm 0.5$ & $5032.2 \pm 0.4$ & $5036.1 \pm 0.5$ \\
\hline
\end{tabular}

Notes. ${ }^{(*)} T_{\mathrm{RV} \max }=$ Time of maximum RV in Heliocentric Julian days -2450000.

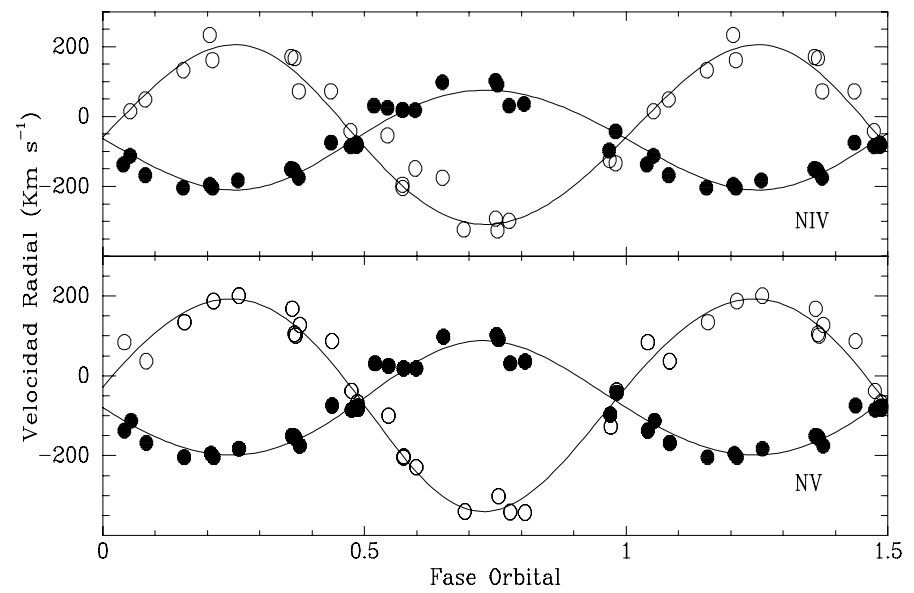

Fig. 4. The RV curves for both components in WR62a. The open circles show the RVs for N IV $\lambda 4058 \AA$ and N V $\lambda 4604 \AA$ emission lines. The filled circles show the mean RVs of some absorption lines for the secondary one. RVs of the emission lines were shifted to match the systemic velocity derived from the absorption lines alone (see text). The ephemeris given in Eq. (1) was used to generate the RV curves.

simultaneous fit of both RV curves, we shifted the emission-line $\mathrm{RV}$ s to match the systemic velocity derived for the O-type component. Therefore, the RVs of N IV and NV were shifted by $80 \mathrm{~km} \mathrm{~s}^{-1}$ and $-86 \mathrm{~km} \mathrm{~s}^{-1}$, respectively (see Table 4 ). These solutions are shown in Table 5 and Fig. 4. Thus, the secondary component is confirmed as the more massive star. Depending on which orbital solution is considered, the mass of the O-type star ranges between 39 and $42 M_{\odot}$, and the WN mass, between 21 and $23 M_{\odot}$. The mass ratio $(q)$ obtained agrees with the values determined in other WN5+OB systems (van der Hucht 2001). The low eccentricity determined using different datasets indicates that the orbit is almost circular, although such a low value should not be neglected.

\subsection{Analysis of spectral features}

In Fig. 5, we show the He II $\lambda 4686$ line at four different orbital phases; the absorption line of the secondary can be seen to be moving in anti-phase with respect to the emission line peak. In some phases, this emission line shows an asymmetric profile, perhaps indicating some additional contribution from the windwind colliding region. We measured the equivalent width (EW) and full-width at half-maximum (FWHM) of the He II $\lambda 4686$, N IV $\lambda$ 44058, and N v $\lambda 4944$ emission lines. We detected a certain variability of these line parameters, which seems to be modulated through the orbit (see Fig. 6). The behaviour of the EW of the He II line is very noticeable: it decreases around phase
Table 5. Orbital solutions for both component of WR 62a

\begin{tabular}{lcc}
\hline \hline & N IV+abs. & N v+abs. \\
\hline$P[\mathrm{~d}]$ & \multicolumn{2}{c}{9.1447 (fixed) } \\
$e$ & $0.05 \pm 0.02$ & $0.05 \pm 0.02$ \\
$K_{\mathrm{WR}}\left[\mathrm{km} \mathrm{s}^{-1}\right]$ & $257 \pm 7$ & $266 \pm 6$ \\
$K_{\mathrm{O}}\left[\mathrm{km} \mathrm{s}^{-1}\right]$ & $143 \pm 6$ & $143 \pm 6$ \\
$V_{0}\left[\mathrm{~km} \mathrm{~s}^{-1}\right]$ & $-62 \pm 3$ & $-62 \pm 3$ \\
$\omega[\mathrm{deg}]$ & $39 \pm 25$ & $149 \pm 20$ \\
$T_{\mathrm{Periast}}[\mathrm{d}]^{*}$ & $5,032.9 \pm 0.6$ & $5,035.7 \pm 0.5$ \\
$T_{\mathrm{RV} \max }[\mathrm{d}]^{*}$ & $5,032.0 \pm 0.6$ & $5,031.9 \pm 0.5$ \\
$a_{\mathrm{WR}} \sin i\left[R_{\odot}\right]$ & $46.1 \pm 1.1$ & $47.8 \pm 1.1$ \\
$a_{\mathrm{O}} \sin i\left[R_{\odot}\right]$ & $25.6 \pm 1.1$ & $25.7 \pm 1.1$ \\
$M_{\mathrm{WR}} \sin ^{3} i\left[M_{\odot}\right]$ & $21.5 \pm 4.8$ & $22.6 \pm 5.0$ \\
$M_{\mathrm{O}} \sin ^{3} i\left[M_{\odot}\right]$ & $38.7 \pm 5.2$ & $42.0 \pm 5.0$ \\
$q_{\mathrm{WR} / \mathrm{O}}$ & $0.56 \pm 0.04$ & $0.54 \pm 0.04$ \\
\hline
\end{tabular}

Notes. ${ }^{(*)}$ HJD-2 450000 .

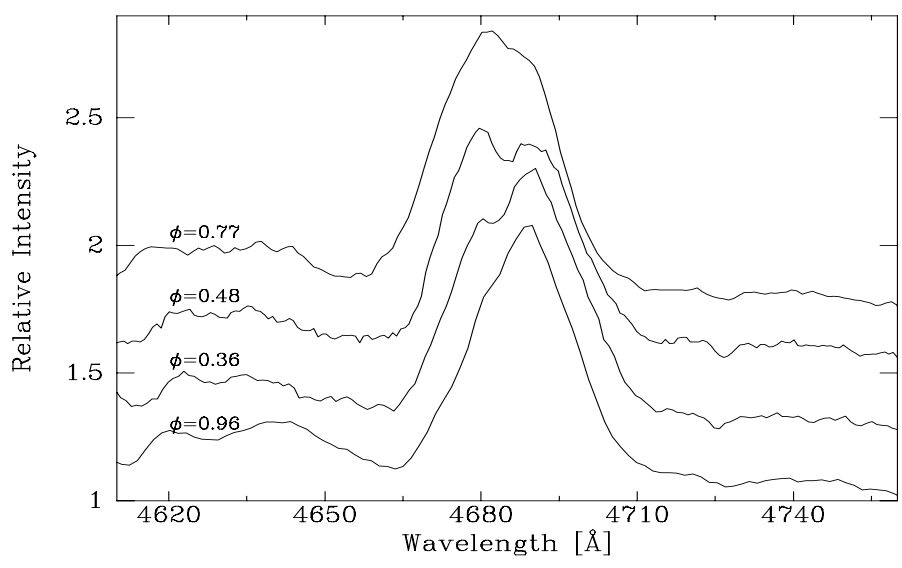

Fig. 5. Continuum rectified spectra of the He II $\lambda 4686$ emission line with the superimposed absorption observed during different orbital phases of the binary. A noticeable anti-phased movement of absorption and emission features is shown.

$\phi=0.0$, when the WN star is in front of the system. This phenomenon is also found in other $\mathrm{WR}+\mathrm{OB}$ binary systems, i.e. WR21 and WR47 (Gamen 2004), and HD 5980 (Moffat et al. 1998), explained as if the emission line had an additional non-stellar component, originating in the colliding-winds region, which is eclipsed during the conjunction phase.

\subsection{Analysis of the available photometry}

As the SB2 orbital solution for WR62a suggests high minimum masses for both components of the system and the EW of the He II $\lambda 4686$ emission line has a minimum at one of the 


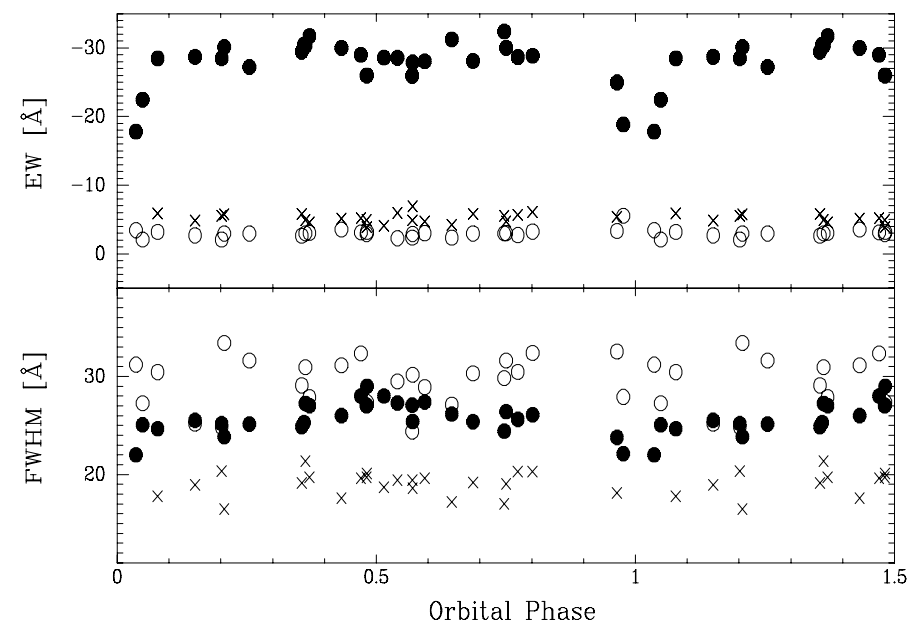

Fig. 6. EW and FWHM measured in the He II $\lambda 4686$ (filled circles), N IV $\lambda 4058$ (crosses), and N V $\lambda 4944$ (open circles) emission lines.

conjunctions, we would expect to observe photometric variability (eclipses, for instance). We, therefore, analysed the data published in the "All-Sky Automated Survey" (ASAS; Pojmański 2001), which gives the $V$ magnitudes for this star. Again, using the code of Marraco \& Muzzio (1980), we searched the ASAS data for periodicities and obtained the most probable period of $9.1438 \pm 0.0009 \mathrm{~d}$, very similar to the period obtained from our RV analysis (see the periodogram in Fig. 7). In Fig. 8, we have plotted the ASAS $V$ magnitudes against the orbital phases generated using the ephemeris given in Eq. (1). We see a V-shaped $\operatorname{dip}$ at $\phi=0.0$. A much shallower dip is marginally detected at $\phi=0.5$. To improve the visualization of the variations, we also show the data points averaged in phase bins of 0.01 in Fig. 8. The shape of the dip at $\phi=0.0$ calls the non-photospheric eclipsing binaries analysed by Lamontagne et al. (1996). In that paper, the authors analysed the eclipsing binary systems WR21 and WR47, where, as we described in the previous sections, the He II $\lambda 4686$ emission line also shows a phase shift in RVs and a minimum of the EW when the WN star passes in the front of the system. The scenario where an additional emission component to the He II $\lambda 4686$ emission is formed in a colliding-wind region, which is being eclipsed by the WN component, should also apply. However, the accuracy of the ASAS data does not allow us to determine the inclination of the system, hence the absolute masses for both components. The $V$ magnitude of WR62a is close to the faint limit of the ASAS survey. More accurate photometry is required to confirm or rule out the eclipsing nature of this binary.

\section{Conclusions}

From our spectroscopic observations and analysis of the RV of the lines detected in the spectra of WR62a, we discovered that it is a double-lined binary system with a $9.1447 \mathrm{~d}$ orbital period. The relative intensities of the emission lines detected in our spectra confirm that the spectral classification of the WN star as WN5, while the absorption lines identified for the secondary star corresponds to an O 5.5-6 spectral-type. The emission lines of $\mathrm{N}, \mathrm{C}$, and He ions originated in the WR star follow the same movement and orbital period, but we detect a slight phase shift in the He II $\lambda 4686$ emission line, which may be due to asymmetries in the line-forming region.

We performed fits of the orbital solution for both components by considering two possible RV datasets of the WN star. From

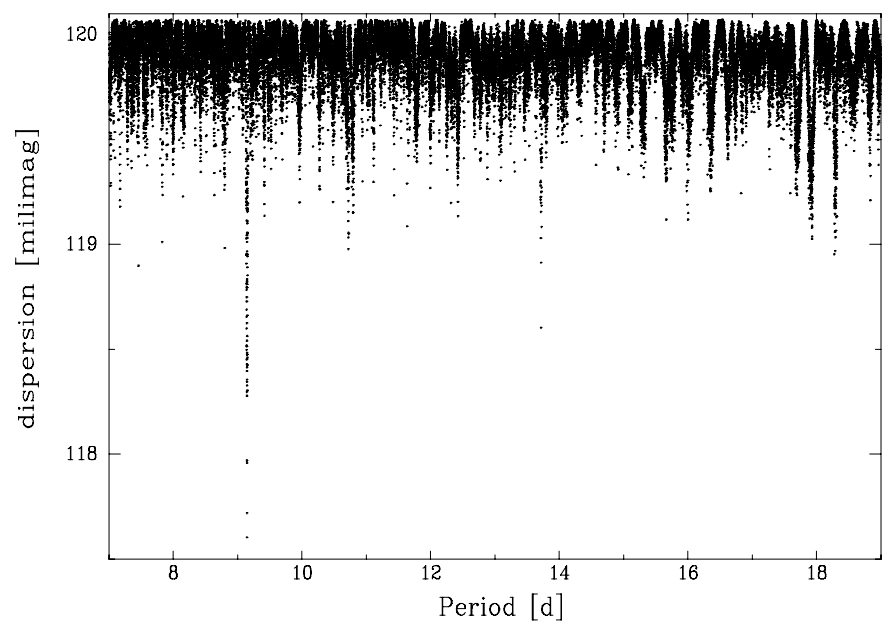

Fig. 7. Periodogram obtained from the ASAS data with the Marraco \& Muzzio (1980) code. The most probable period found is $9.1438 \pm 0.0009 \mathrm{~d}$.

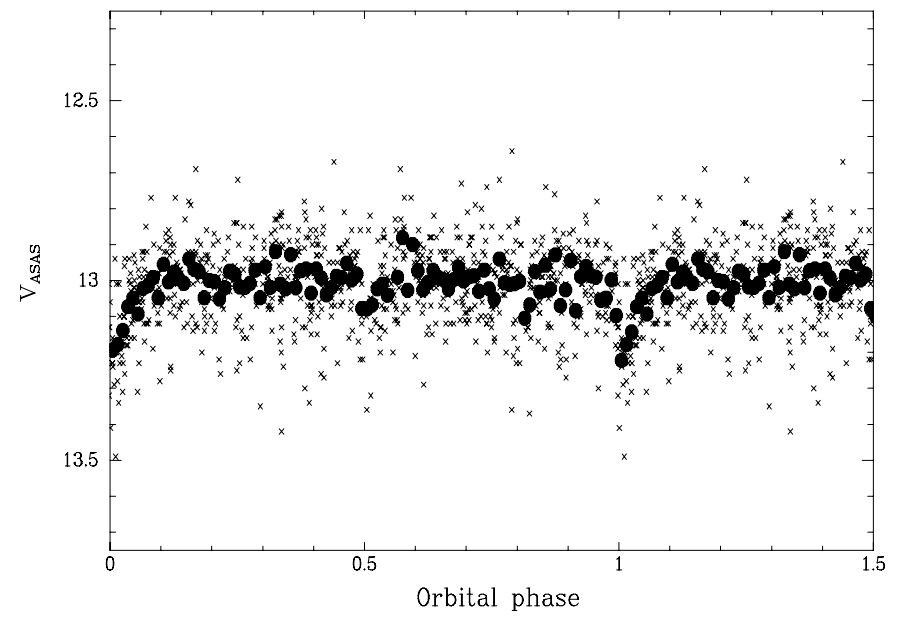

Fig. 8. The lightcurve of WR62a using the ASAS data. The individual data-points are shown by crosses; the filled circles represent the datapoints averaged in phase bins of 0.01 . The ephemeris derived from our spectroscopic data (Eq. (1)) is used to calculate the orbital phases.

these solutions, we confirm that the O-type star is more massive than the WN star. The minimum mass derived for the O-type star ranges from 39 to $42 M_{\odot}$, and for the WN-type star from 21 to $23 M_{\odot}$, depending on which emission line is used to represent the orbital motion of the WN star, i.e. N IV $\lambda 4058$ or N V $\lambda 4604$ respectively.

We detected variations in the EW of the He II $\lambda 4686$ emission line with the orbital period, showing a $\sim 30 \%$ "dip" around $\phi=0$, where the WN star is in front. This effect suggests that an additional emission component to this line, originated in a collidingwind zone, is being eclipsed. Analysing photometric data from ASAS, we also identified a minimum when the WN star is in front of the system. Additionally, a very marginal secondary dip is detected in the opposite orbital phase (around $\phi=0.5$ ). The quality of ASAS data does not allow us to determine the inclination of the system hence the absolute masses for both components. To confirm the eclipsing nature of WR62a, we need more accurate photometry.

The spectrum of WR62a shows a remarkable resemblance to the WR+O binary system WR21 (Gamen 2004). Similar phenomena are found in both binary systems. These systems could be used as test benches for geometric colliding-wind models. 
A. Collado et al.: The new Wolf-Rayet binary system WR62a

Acknowledgements. We are very grateful to the referee for the constructive comments We thank the directors and staff of CASLEO, LCO, and CTIO for allowing us the use of their facilities. We especially thank Nidia "Hada" Morrell for kindly obtaining some spectra.

\section{References}

Barbá, R. H., Gamen, R., Arias, J. I., et al. 2010, in Rev. Mex. Astron. Astrofis. Conf. Ser., 38, 30

Bertiau, F., \& Grobben, J. 1968, Ric. Astr. Spec. Vat., 8, 1

Gamen, R. 2004, Ph.D. Thesis, La Plata University

Lamontagne, R., Moffat, A. F. J., Drissen, L., Robert, C., \& Matthews, J. M. 1996, AJ, 112, 2227

Langer, N., \& Heger, A. 1999, in Wolf-Rayet Phenomena in Massive Stars and Starburst Galaxies, eds. K. A. van der Hucht, G. Koenigsberger, \& P. R. J. Eenens, IAU Symp., 193, 187
Marchenko, S. V., Moffat, A. F. J., \& Eenens, P. R. J. 1998, PASP, 110, 1416 Marraco, H., \& Muzzio, J. 1980, PASP, 92, 700

Meynet, G., \& Maeder, A. 2005, A\&A, 429, 581

Moffat, A. F. J., Marchenko, S. V., Bartzakos, P., et al. 1998, ApJ, 497, 896

Niemela, V. S., \& Moffat, A. 1982, Ap.J., 259, 213

Niemela, V. S., Cabanne, M. L., \& Bassino, L. P. 1995, Rev. Mex. Astron. Astrofis., 31, 45

Pojmański, G. 2001, in IAU Colloq. 183, Small Telescope Astronomy on Global Scales, eds. B. Paczynski, W.-P. Chen, \& C. Lemme, ASP Conf. Ser., 246, 53 Sana, H., \& Evans, C. J. 2011, in IAU Symp. 272, eds. C. Neiner, G. Wade, G. Meynet, \& G. Peters, 474

Shara, M. M., Moffat, A. F. J., Smith, L. F., et al. 1999, AJ, 118, 390

Smith, L., Shara, M., \& Moffat, A. 1996, MNRAS, 281, 163

Sota, A., Maíz Apellániz, J., Walborn, N. R., et al. 2011, ApJS, 193, 24

Usov, V. V. 1991, MNRAS, 252, 49

van der Hucht, K. A. 2001, New Astron. Rev., 45, 135 